\title{
Spectroscopic neutron detection using composite scintillators
}

\author{
I. Jovanovic*, A. Foster, V. Kukharev, M. Mayer,A. Meddeb. J. Nattress, Z. Ounaies and C. Trivelpiece \\ The Pennsylvania State University, 228 Reber Building, \\ University Park, Pennsylvania 16802, United States \\ *ijovanovic@psu.edu
}

Published 1 September 2016

\begin{abstract}
Shielded special nuclear material (SNM), especially highly enriched uranium, is exceptionally difficult to detect without the use of active interrogation (AI). We are investigating the potential use of low-dose active interrogation to realize simultaneous high-contrast imaging and photofission of SNM using energetic gamma-rays produced by low-energy nuclear reactions, such as ${ }^{11} \mathrm{~B}(\mathrm{~d}, \mathrm{n} \gamma)^{12} \mathrm{C}$ and ${ }^{12} \mathrm{C}\left(\mathrm{p}, \mathrm{p}^{\prime}\right)^{12} \mathrm{C}$. Neutrons produced via fission are one reliable signature of the presence of SNM and are usually identified by their unique timing characteristics, such as the delayed neutron die-away. Fast neutron spectroscopy may provide additional useful discriminating characteristics for SNM detection. Spectroscopic measurements can be conducted by recoil-based or thermalization and capture-gated detectors; the latter may offer unique advantages since they facilitate low-statistics and event-by-event neutron energy measurements without spectrum unfolding. We describe the results of the development and characterization of a new type of capture-gated spectroscopic neutron detector based on a composite of scintillating polyvinyltoluene and lithium-doped scintillating glass in the form of millimeter-thick rods. The detector achieves $>10^{8}$ neutron-gamma discrimination resulting from its geometric properties and material selection. The design facilitates simultaneous pulse shape and pulse height discrimination, despite the fact that no materials intrinsically capable of pulse shape discrimination have been used to construct the detector. Accurate single-event measurements of neutron energy may be possible even when the energy is relatively low, such as with delayed fission neutrons. Simulation and preliminary measurements using the new composite detector are described, including those conducted using radioisotope sources and the low-dose active interrogation system based on low-energy nuclear reactions.
\end{abstract}

Keywords: special nuclear material; scintillation detector; capture gating.

\section{Introduction: Fast Neutron Detection in Active Interrogation}

Detection of special nuclear material (SNM), especially when shielded, is an exceptionally challenging problem in nuclear security. The relatively low rate of emission of characteristic penetrating radiation (gammas and neutrons) can be further reduced by a relatively small thickness of shielding. Active interrogation by an external source of penetrating radiation is needed to perform radiography of a suspected object and to simultaneously enhance the rate of characteristic radiation emission by fission.

This is an Open Access article published by World Scientific Publishing Company. It is distributed under the terms of the Creative Commons Attribution 3.0 (CC-BY) License. Further distribution of this work is permitted, provided the original work is properly cited. 
Several active interrogation methods have been proposed and have undergone testing to date. Of those, photon-based interrogation offers the prospect of lower radiation dose delivered to the scanned object. In bremsstrahlung-based systems, an electron beam impinges on a target and produces a continuum of X-ray radiation, whereby the lowerenergy component of the X-ray spectrum contributes to the radiation dose but cannot contribute to the useful signal needed for inspection. Monoenergetic sources of photons, such as those based on low-energy nuclear reactions and inverse Compton scattering could significantly reduce the radiation dose, thereby improving the prospects for their practical application.

We study the use of the ${ }^{11} \mathrm{~B}(\mathrm{~d}, \mathrm{n} \gamma){ }^{12} \mathrm{C}$ nuclear reaction, producing $4.4 \mathrm{MeV}$ and 15.1 $\mathrm{MeV}$ gamma rays and simultaneously energetic neutrons as a prospective technology for active interrogation of shielded SNM. In addition to dual-energy photon and neutron radiography that this source enables, ${ }^{1}$ the fission induced by energetic $(15.1 \mathrm{MeV})$ photons and neutrons can also be used to generate the unique signature of the presence of SNM. Specifically, one promising signature of fission we are studying is the emission of beta-delayed neutrons, which are emitted over timescales of seconds to minutes and exhibit very low background. However, their abundances and average energies $(\sim 0.5$ $\mathrm{MeV}$ ) are low, and they are immersed in a complex background of beta-delayed gammas and activation gammas.

Detection of these low-energy delayed neutrons thus requires detectors that have the exceptional capability to discriminate neutrons from gammas at low energies and that can be scaled up to achieve large coverage at low specific cost. We also desire detectors that retain spectroscopic information, which can aid the understanding of the material shielding and be used in conjunction with fast neutron radiography. We have identified composite polymer-glass scintillators as a promising technology to achieve those detection goals. We summarize our recent progress in designing and testing novel composite scintillators for application in active interrogation, with special attention given to their spectroscopic capabilities.

\section{Principle of Operation of a Composite Scintillation Detector}

The operational principle of a composite scintillator (see Fig. 1) is based on the combined use of scintillation materials with varying fluorescence decay time constants, large differences of cross-sections for different particle types, and differences in the typical range of charged particles produced in the neutron and gamma interactions. Individually, each scintillating material does not possess pulse shape discriminating (PSD) capabilities. In a suitably designed composite, a gamma interacts primarily through Compton scattering, producing recoil electrons, whereas a neutron interacts through a combination of elastic scattering (at high energies) and capture (at thermal energies), producing heavy charged particles with energies determined by the Q-value of the neutron capture reaction. The amplitude and the shape of the resulting scintillation pulse are dependent on the location of interaction and the path traversed by the produced charged particle. By constricting geometrical dimensions and careful material selection, discrimination 
between neutrons and gammas can be achieved through both pulse height and shape analysis. ${ }^{2,3}$

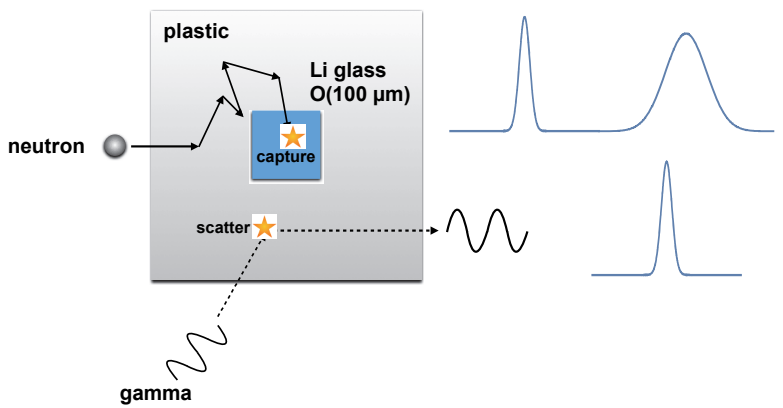

Fig. 1. Principle of operation of a composite scintillation detector exposed to fast neutrons and gamma rays.

We use the lithium-doped glass GS20 in the form of thin, long rods as the thermal neutron detector component and scintillating polyvinyltoluene (PVT) from Eljen (EJ290) as the moderator component of the detector (Fig. 2). Lithium-doped glass in the form of rods is commercially available, while the use of PVT permits relatively easy fabrication into a variety of shapes.

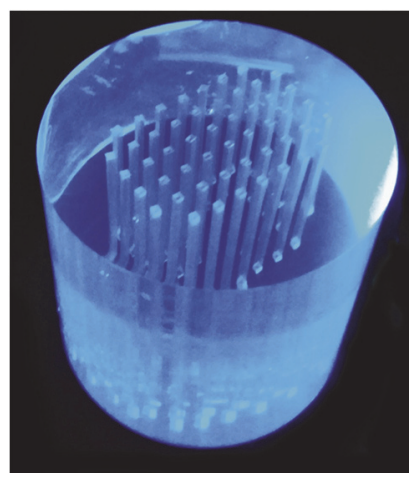

Fig. 2. Composite neutron detector used in simulation and experiments ( $50 \mathrm{~mm}$ diameter, $50 \mathrm{~mm}$ height).

${ }^{6} \mathrm{Li}$ has a high thermal neutron capture cross-section of approximately $940 \mathrm{~b}$ and a high Q-value compared to the competing energy depositions of recoil electrons produced in typical gamma interactions, making it an attractive choice for use in a composite detector. The choice of scintillating PVT allows the thermalization energy of the neutron to be measured, which could facilitate capture-gated fast neutron spectrometry.

\section{Detector Simulation}

The design and simulation of our detector architecture using the Geant 4 framework has been previously described in detail. ${ }^{2}$ Here we provide additional results relevant for detector scaling and neutron spectroscopy. The predicted capture efficiency for prompt neutrons produced by the ${ }^{252} \mathrm{Cf}$ source for a $50 \mathrm{~mm}$ diameter detector with a $6 \%$ weight 


\section{Jovanovic et al.}

ratio of GS20 glass to PVT, which was constructed for this study, is $0.49 \%$. Simulations further predict that the efficiency should increase by one to two orders of magnitude as the detector diameter is increased by a factor of two to three and the GS20 to PVT ratio is increased to $7 \%$. The increase in size is needed to make the detector suitable for detection of the relatively low rate of beta-delayed neutrons. Since the required increase in size is relatively modest, it is not expected that the optical light transport efficiency will be significantly affected.

In order to realize neutron spectroscopy in the composite detector, it is necessary to measure the scintillation light produced by neutron thermalization in the PVT component of the detector. The simulated distribution of the thermalization-to-capture time in our detector design is shown in Fig. 3. The probability of neutron capture is high only if the neutron is fully thermalized in the PVT. Therefore, if the neutron capture pulse is present, the preceding thermalization pulse is due to full energy deposition of the incoming neutrons. Depending on the linearity of the response of the thermalizing component of the detector, event-by-event spectroscopy may be possible even without spectral unfolding, which is normally required for recoil-based fast neutron detectors. The correlation between the scintillation output of PVT and the energy of the incoming neutron needs to be measured to further investigate the merit of this approach.

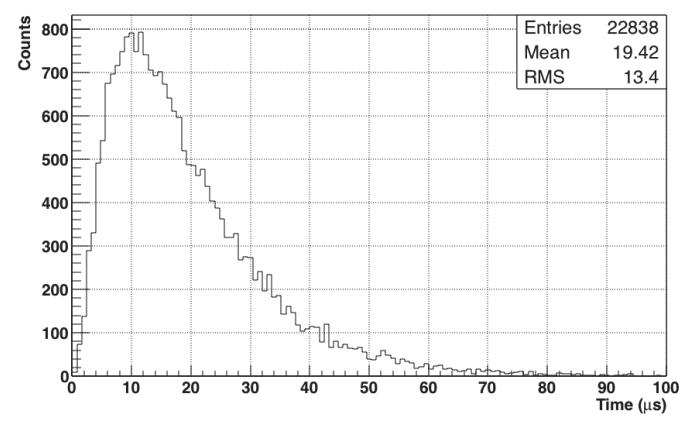

Fig. 3. Calculated thermalization-to-capture time in composite detector.

\section{Detector Testing with Radioisotope Sources}

The ${ }^{252} \mathrm{Cf}$ neutron source used for detector characterization had an activity of $0.83 \mathrm{mCi}$, providing $3.6 \times 10^{6}$ neutrons/s. The experiment was conducted in an isolated room that had a concrete floor, which provides an abundance of room-return neutrons, significantly increasing the incident flux of neutrons on the detector and modifying the incident neutron spectrum. A shadow cone was constructed and used to subtract the room-return neutrons, as shown in Fig. 4. The source-to-detector distance was set to $1.4 \mathrm{~m}$. Measurements were completed with and without the shadow cone for one hour each and subtracted. The data acquisition window was optimized to contain both the thermalization and capture pulses, as in Fig. 5. 


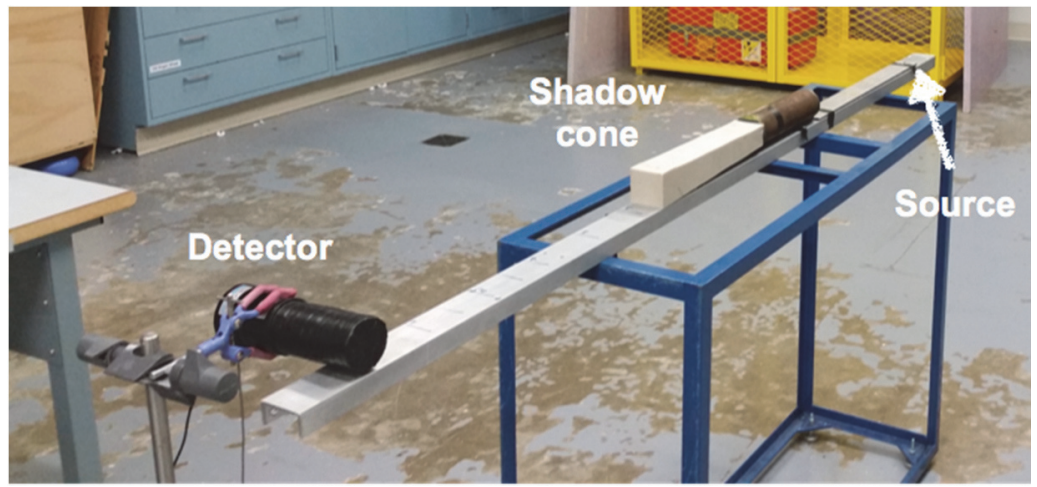

Fig. 4. Detector test configuration.

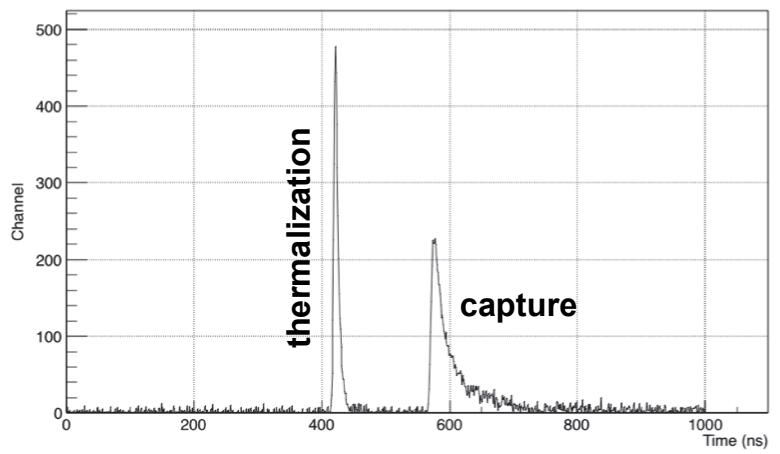

Fig. 5. A typical thermalization-capture event in the composite detector.

A full and partial integration was performed for each pulse to define the pulse shape parameter (PSP), defined in the standard fashion as the ratio of tail to full integral of the pulse. The partial integration (tail) started 14 ns following the peak. A neutron event was identified by the presence of a pronounced tail region, which is characteristic of scintillation in the glass component of the detector. Figure 6 shows the localized event region corresponding to neutron capture in an energy range of approximately 400-600 $\mathrm{keV}$ and centered at $\mathrm{PSP}=0.6$, as well as the band constrained to approximately $0.1<\mathrm{PSP}<0.3$, which contains both the neutron recoils and the electron recoils that deposit energy primarily in the PVT. A double Gaussian was fit to the neutron capture region, and events within $3 \sigma$ are attributed to neutron captures. These cuts resulted in an intrinsic neutron capture efficiency of $0.33 \%$ and gamma rejection exceeding $10^{8}$, as previously reported. ${ }^{3}$ 


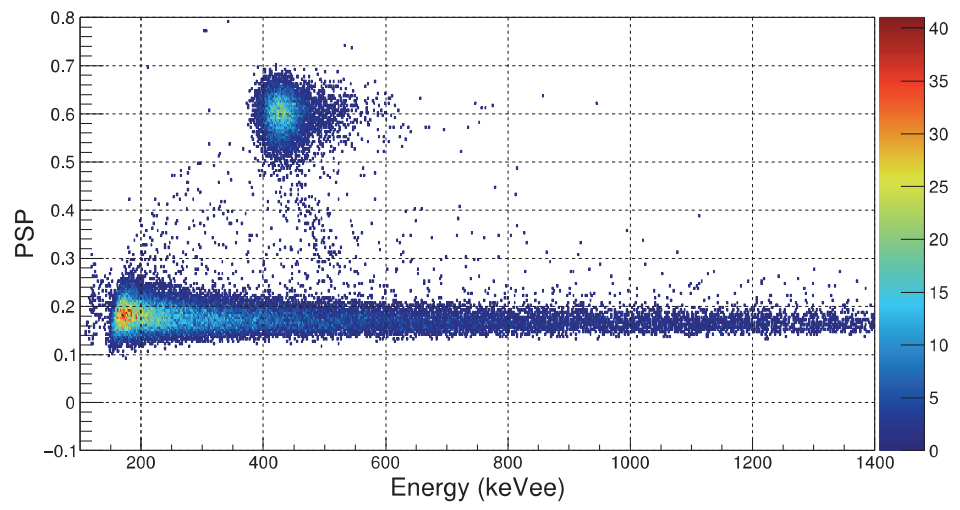

Fig. 6. Pulse shape discrimination characteristic measured using a ${ }^{252} \mathrm{Cf}$ source.

The capture-gated scintillation spectrum was obtained by identifying a neutron event and accepting the previous pulse. Figure 7 shows the obtained capture-gated scintillation spectrum from neutrons directly incident on the detector from the ${ }^{252} \mathrm{Cf}$ source. Future experiments are set to measure the composite's response to monoenergetic neutron sources in a low neutron return environment using DD and DT generators and a tagged ${ }^{252} \mathrm{Cf}$ source. Results from these measurements will provide results to further develop an understanding of the spectroscopic capabilities of the composite neutron detector.

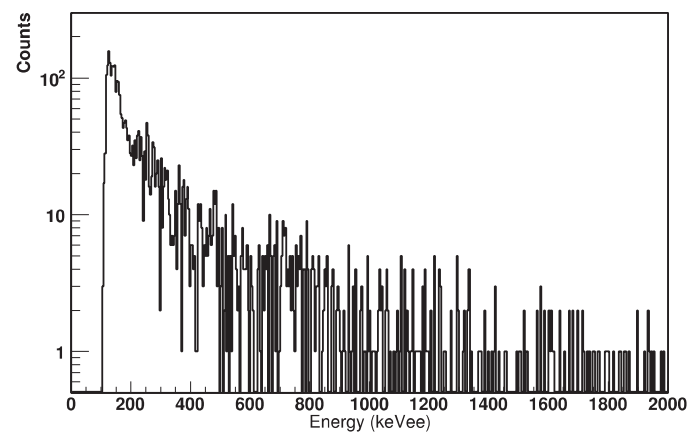

Fig. 7. Capture-gated spectrum measured using a ${ }^{252} \mathrm{Cf}$ source.

\section{Preliminary Detector Tests in an Active Interrogation Environment}

We conducted preliminary detector tests in an active interrogation environment provided by a ${ }^{11} \mathrm{~B}(\mathrm{~d}, \mathrm{n} \gamma){ }^{12} \mathrm{C}$ source at the MIT Bates Accelerator Laboratory. In these measurements, the neutrons produced directly by the ${ }^{11} \mathrm{~B}$ target were shielded by borated polyethylene. The gamma rays were collimated to a fan beam and directed to a variety of objects composed of different materials, such as natural $U$ and $W$. The estimated 15.1 MeV gamma yield produced at the ${ }^{11} \mathrm{~B}$ target was of the order $10^{8} / \mathrm{s}$, and the distance between the ${ }^{11} \mathrm{~B}$ target and the objects was approximately $2 \mathrm{~m}$. The composite detector was positioned in close proximity to the object, but outside of the fan beam. 


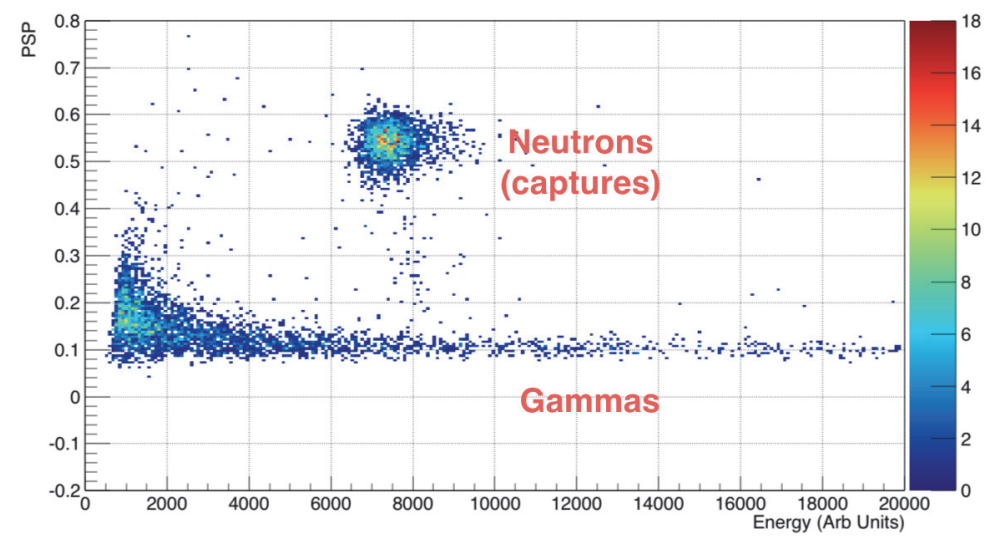

Fig. 8. Pulse shape discrimination characteristic measured in active interrogation environment provided by the ${ }^{11} \mathrm{~B}(\mathrm{~d}, \mathrm{n} \gamma){ }^{12} \mathrm{C}$ nuclear reaction-based source.

As shown in Fig. 8, we found excellent separation between neutrons and gamma rays in this complex radiation environment. The distinction between neutrons and gammas is comparable to that measured using the ${ }^{252} \mathrm{Cf}$ radioisotope source. We found no significant difference in detected prompt neutron rates between the measurements conducted on material undergoing fission (U) and other high-Z material, such as $\mathrm{W}$. This can be explained by the comparable cross sections for photoneutron production by the $15.1 \mathrm{MeV}$ gamma ray for high- $Z$ material, which exceed the photofission cross section of uranium. It is thus unlikely that discrimination can be made between SNM and other high-Z materials based on prompt neutron production rates, and delayed neutrons should be used as a signature radiation specific to SNM.

\section{Conclusion}

In conclusion, we developed a novel type of composite scintillator based on scintillating PVT and scintillating Li-doped glass which takes advantage of the physics of interaction and energy deposition in an optimized composite configuration to realize an exceptional level of gamma rejection $\left(>10^{8}\right)$, even at low incident neutron energies. This characteristic makes the detector an attractive candidate for detecting beta-delayed neutrons as a unique signature of SNM in active interrogation. We found that the neutron-gamma discrimination was not compromised in the complex active interrogation environment provided by a ${ }^{11} \mathrm{~B}(\mathrm{~d}, \mathrm{n} \gamma)^{12} \mathrm{C}$ nuclear reaction-based source.

The experimental neutron capture efficiency was $0.33 \%$, in good agreement with simulation, and simulations predict that the efficiency can be increased by approximately two orders of magnitude by increasing the present detector size $(50 \mathrm{~mm}$ diameter $\mathrm{x} 50$ $\mathrm{mm}$ height) by a factor of three. The promising preliminary measurements of the thermalization light output during a neutron capture event offer the prospect of event-byevent spectroscopy that may not require the conventional spectral unfolding that is characteristic of recoil-based detectors. Additional measurements are needed to explore 
the correlation of the thermalization light yield and incident neutron energy at a variety of neutron energies.

\section{Acknowledgments}

This research was funded by the National Science Foundation and the Domestic Nuclear Detection Office of the Department of Homeland Security through the Academic Research Initiative Award ECCS-1348328.

\section{References}

1. R. C. Lanza, A. Erickson, J. Fisher, I. Jovanovic, et al., Nuclear reaction based monoenergetic gamma ray radiography system for detection of nuclear materials, in Nuclear Science Symposium and Medical Imaging Conference (NSS/MIC), (2014).

2. M. Mayer, J. Nattress, C. Trivelpiece, and I. Jovanovic, Nucl. Instr. Meth. Phys. Res. A 784, 168 (2015).

3. M. Mayer, J. Nattress, V. Kukharev, A. Foster, A. Meddeb, C. Trivelpiece, Z. Ounaies, and I. Jovanovic, Nucl. Instr. Meth. Phys. Res. A 785, 117 (2015). 